\title{
Extrauterine high-grade serous carcinomas with bilateral adnexal involvement as the only two disease sites are clonal based on tp53 sequencing results: implications for biology, classification, and staging
}

\author{
Naveena Singh ${ }^{1}$, Asma Faruqi ${ }^{1}$, Friedrich Kommoss ${ }^{2,3}$, W Glenn McCluggage ${ }^{4}$, \\ Giorgia Trevisan ${ }^{1}$, Janine Senz ${ }^{3}, \mathrm{Amy} \mathrm{Lum}^{3}$, C Blake Gilks ${ }^{2,3}$ and Michael Anglesio ${ }^{3}$ \\ ${ }^{1}$ Department of Cellular Pathology, Barts Health NHS Trust, London, UK; ${ }^{2}$ Department of Pathology and \\ Laboratory Medicine, Vancouver General Hospital, Vancouver, BC, Canada; ${ }^{3}$ British Columbia Cancer \\ Agency, Molecular Oncology, Vancouver, BC, Canada and ${ }^{4}$ Department of Pathology, Belfast Health and \\ Social Care Trust, Northern Ireland, UK
}

\begin{abstract}
A previous multicenter study of 67 cases of Stage I/I tubo-ovarian high-grade serous carcinoma with complete tubal sampling identified 7 cases in which there were only two disease sites, comprising tumor involving opposite adnexa with no extra-adnexal involvement. This study aimed to determine whether such low-stage extrauterine high-grade serous carcinomas with only two sites of involvement, located on opposite adnexa, have identical or different TP53 mutations in order to investigate their clonal relationship. DNA extracted from both sites of involvement was subjected to TP53 sequencing $(n=6)$ or sequencing of one site and mutation confirmation by droplet digital PCR for the other site $(n=1)$. Of the 7 cases analyzed, 1 case had unilateral serous tubal intraepithelial carcinoma with contralateral ovarian high-grade serous carcinoma, 3 had tubal high-grade serous carcinomas ( \pm serous tubal intraepithelial carcinoma) with contralateral ovarian high-grade serous carcinoma, 2 had bilateral ovarian high-grade serous carcinomas with normal tubes, and 1 had bilateral fallopian tube high-grade serous carcinoma with normal ovaries. All 7 cases showed identical TP53 mutations in tumor from both disease sites. Therefore, these rare cases of high-grade serous carcinoma confined to opposite adnexa all show clonal identity between the two sites of involvement, suggesting unifocal origin and metastasis rather than multifocal origin. Our results suggest that serous tubal intraepithelial carcinoma or adnexal highgrade serous carcinoma can metastasize to the contralateral adnexa without peritoneal involvement. Given the clonal relationship between the two sites, such cases should be considered stage II, with stage I reserved for cases with unilateral and unifocal adnexal involvement. Furthermore, serous tubal intraepithelial carcinoma without invasion should be taken to constitute a disease site for staging purposes.

Modern Pathology (2018) 31, 652-659; doi:10.1038/modpathol.2017.159; published online 17 November 2017
\end{abstract}

The theories regarding the development of extrauterine-high grade serous carcinoma have included origin from the ovarian surface epithelium, ${ }^{1}$ from cortical inclusion cysts, ${ }^{2}$ from the pelvic peritoneum in cases where there is minimal or no ovarian involvement (primary

Correspondence: Dr N Singh, MD, FRCPath, Department of Cellular Pathology, Barts Health NHS Trust, 2nd Floor, 80 Newark Street, London E1 2ES, UK.

E-mail: N.Singh@bartshealth.nhs.uk

Received 15 August 2017; revised 30 September 2017; accepted 7

October 2017; published online 17 November 2017 peritoneal high-grade serous carcinoma), ${ }^{3}$ and multifocal origin within a field change. ${ }^{4-6}$ In the past 10 years, however, the theory of origin from serous tubal intraepithelial carcinoma of the fallopian tube epithelium has gained wide acceptance. ${ }^{7,8}$ The SEEFIM (Sectioning and Extensively Examining the FIMbriated end of the fallopian tube) protocol, with serial sectioning and submission of the entire fallopian tube, is now the standard of care in the pathological examination of extrauterine high-grade serous carcinoma, especially early-stage tumors. This practice has resulted in the identification of serous tubal intraepithelial carcinoma or small tubal 
mucosal high-grade serous carcinoma in a significant number of cases of sporadic high-grade serous carcinoma and has resulted in further understanding of the relationship between serous tubal intraepithelial carcinoma and high-grade serous carcinoma. ${ }^{9-11}$ It can be summarized that there is accumulating and convincing evidence that most extrauterine highgrade serous carcinomas, both sporadic and hereditary, arise from the fallopian tube, with impact on disease classification. ${ }^{12-16}$

Although most extrauterine high-grade serous carcinomas present at advanced stage, that is, stage III or IV, the minority of cases where tumor is confined to the pelvis offer unique insights into the earliest events in oncogenesis. In recent studies of incidentally detected high-grade serous carcinoma in non-high-risk women, all examples with a single site of tumor involvement were located in the fallopian tube mucosa, ${ }^{9-11}$ arguably the strongest evidence supporting the tubal origin of extrauterine highgrade serous carcinoma in most cases. In this study, we examined cases selected based on the very uncommon scenario of the presence of high-grade serous carcinoma/serous tubal intraepithelial carcinoma involving only two anatomic sites, specifically with bilateral adnexal involvement. Our objective was to assess clonality in these cases based on comparison of TP53 mutations, and thereby to investigate whether these separate lesions on opposite adnexa were more likely to represent primary and metastatic tumor, or independent tumors arising from multifocal origin within a cancerized field.

\section{Materials and methods}

\section{Study Cohort and Procedures}

A previously reported series of 67 cases of FIGO stage I and II extrauterine high-grade serous carcinoma, ${ }^{17}$ in which the tubes had been sampled entirely according to the SEE-FIM protocol, revealed 7 cases with only two sites of disease, specifically located on opposite adnexa. This was found to be a rare occurrence $(10 \%)$ in low-stage cases. Blocks were retrieved for molecular studies from 6 of these 7 cases, and from one additional case reported after the initial study.

\section{Tumor Enrichment and DNA Extraction}

Serial sections of formalin-fixed, paraffin embedded tissue were made and one stained with hematoxylin and eosin. In 5 cases (cases 1-5), sufficient tumor was present in both foci to allow for sampling of the whole section, or manual macrodissection of unstained paraffin sections. In these cases, DNA was extracted using the QiaAMP formalin-fixed, paraffin embedded tissue DNA extraction kit (Qiagen). In the remaining two cases (cases 6 and 7) one focus was enriched by manual macrodissection of unstained paraffin sections (and DNA extracted as noted above), and the other focus was enriched through needle macrodissection of hematoxylin and eosin-stained sections (case 6) or laser-capture microdissection of hematoxylin and eosin-stained sections on an LMD7000 (Leica) laser microdissection microscope (case 7). The latter two specimens had DNA extracted using the Pico-Pure DNA extraction kit (Thermo-Fisher).

\section{TP53 Sequencing}

DNA from all cases (except the serous tubal intraepithelial carcinoma from case 7 , see below) was subjected to amplicon-based next-generation sequencing $^{18}$ of the entire TP53 gene (Supplementary Table S1 for primer sequences). Sufficient DNA was available for orthogonal validation of variants, in both tumor foci in cases 1-5 and the larger lesions from cases 6 and 7, by Sanger sequencing. The serous tubal intraepithelial carcinoma focus for case 7 was too small to allow for nextgeneration sequencing but sufficient DNA was recovered for droplet digital PCR confirming the presence of the same TP53 alteration observed in the contralateral ovarian high-grade serous carcinoma.

\section{Droplet Digital PCR}

High-resolution droplet digital PCR with was performed using 5 ' exonuclease assays ('TaqMan') with allele-specific dual-labeled probes corresponding to mutant c.743G $>$ A (FAM reporter) and wild-type (HEX reporter) TP53 alleles (BioRad Laboratories; mutant assay dHsaCP2000107; wild-type assay dHsaCP2000108), both with nonfluorescent quencher. Hundreds of thousands of water-oil emulsion microdroplet PCR reactions were generated, each with a single template DNA molecule using the QX200 Droplet Generator (BioRad Laboratories). Emulsion droplets were subject to thermal cycling in a standard thermal cycler (Bio-Rad Tetrad 2) and, when bound, allelespecific probes were degraded by exonuclease activity of Taq, releasing the $5^{\prime}$ reporter from the $3^{\prime}$ quencher and resulting in fluorescence (normally suppressed by FRET). Fluorescence of the reporter channels was measured and plotted (Figure 2). Digital counting of the number of mutant (FAM) or wild-type (HEX) fluorescing drops was performed with the QX200 Droplet Reader (Bio-Rad Laboratories) and directly representative of the allelic representation in the sample. The high count number and digital nature of the assay allow for exceptionally high sensitivity detection of mutant alleles.

\section{Results}

Details of the 7 cases are shown in Table 1 . The patients ranged in age from 45 to 65 years. Of the 7 
cases, 1 patient had unilateral serous tubal intraepithelial carcinoma with contralateral ovarian highgrade serous carcinoma, 3 had tubal high-grade serous carcinoma ( \pm serous tubal intraepithelial carcinoma) with contralateral ovarian high-grade serous carcinoma, 2 had bilateral ovarian highgrade serous carcinoma with normal tubes, and 1 had bilateral fallopian tube high-grade serous carcinoma with normal ovaries. In accordance with inclusion criteria, there were no other sites of disease in these women, all of whom had been surgically staged. The sizes of the tubal high-grade serous carcinomas ranged from 7 to $140 \mathrm{~mm}$, and the ovarian high-grade serous carcinomas ranged from 3 to $150 \mathrm{~mm}$. All 7 cases showed abnormal mutationtype p53 expression on immunohistochemistry; this was a diffuse nuclear overexpression pattern in 6 cases and a complete absence ('null') pattern in one case. In the 6 cases with overexpression, the immunostaining pattern was identical in both tumor foci (Figure 1). For the remaining case with a 'null' pattern in one ovary, there was insufficient residual tumor in the block from the contralateral ovary, following TP53 sequencing, to allow confirmation of a concordant pattern of expression.

Identical TP53 mutations were seen in the paired contralateral adnexal tumors of all seven cases, as shown in Table 1. The serous tubal intraepithelial carcinoma lesion in case 7 gave a unique opportunity to examine what appeared in two dimensions, on the glass slide, as three distinct foci in the tubal fimbria (Figure 2). Each was laser-capture microdissected individually and tested for the presence of the c.742C > T (p.W248R) variant observed in the contralateral ovarian high-grade serous carcinoma, using droplet digital PCR. All three foci were positive for the mutation.

\section{Discussion}

This is the first study to investigate the clonal relationship between sites of involvement by 'early' extrauterine high-grade serous carcinoma, confined to two sites on opposite adnexa. Based on the finding of identical TP53 mutations, our results demonstrate that these cases are clonal, a finding in keeping with the results of many previous studies that have demonstrated identical TP53 mutations in serous tubal intraepithelial carcinoma and disseminated high-grade serous carcinoma., 19-23 TP53 mutation is an early and almost ubiquitous molecular event in extrauterine high-grade serous carcinoma. ${ }^{24,25}$ As mutations in TP53 are not confined to hot spots, but are spread throughout the gene, the finding of identical somatic TP53 mutations in high-grade serous carcinoma at two anatomic sites is compelling evidence that these are clonally related; in these early lesions, it also suggests that one site represents the primary tumor and the other a metastasis. Thus, there is no evidence to support the multifocal origin

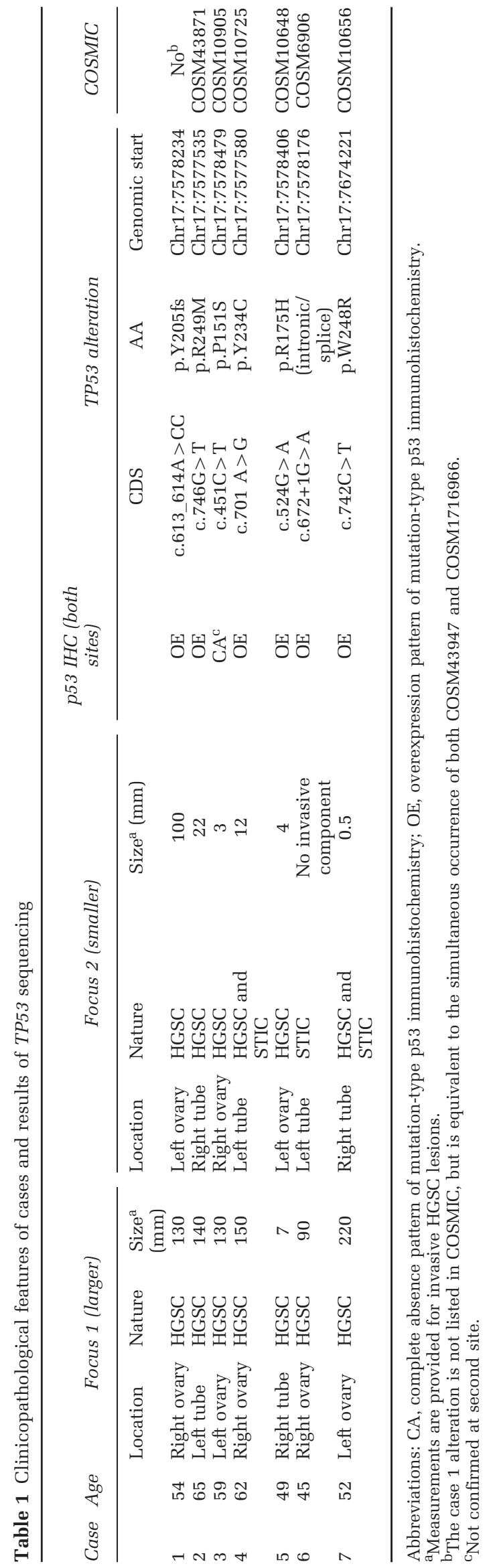




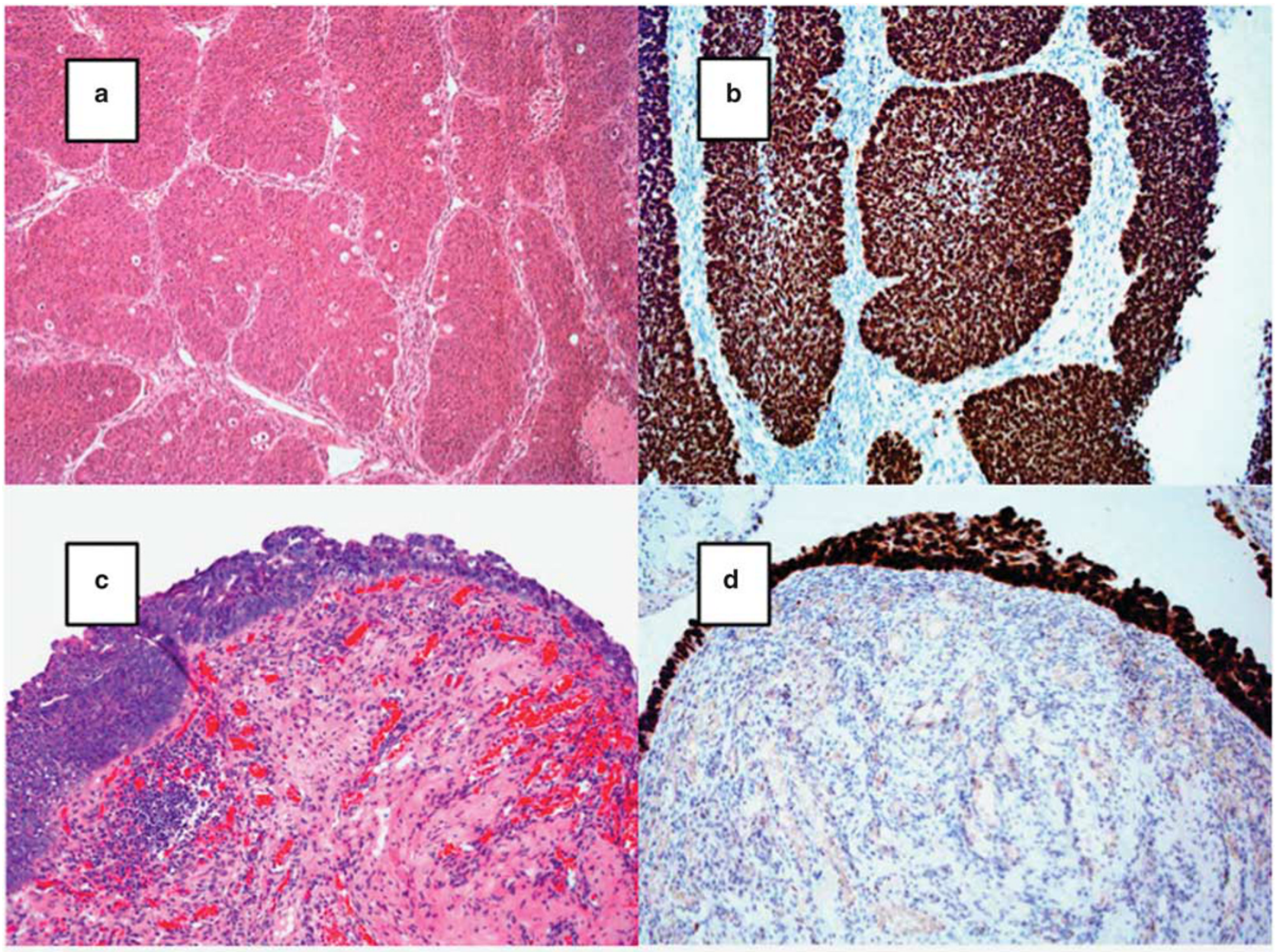

Figure 1 A case of STIC (a) and contralateral HGSC (c) with corresponding concordant mutation-type p53 immunohistochemistry (b, d).

of extrauterine high-grade serous carcinoma development (extremely rare exceptions in high-risk patients are discussed below). The p53 immunohistochemical staining results were also identical at the two sites of involvement, with all six cases where this could be evaluated showing a concordant mutation-type overexpression pattern.

The concept of 'field cancerization' (also known as field effect/change) introduced in 1950s is well established, though the mechanisms by which cancer might develop from a population of normal cells remain poorly understood. ${ }^{26,27}$ Field cancerization comprises a host of mechanisms that predispose an entire region of susceptible cells to the development of cancer through the acquisition of sequential genomic alterations. There is a need for more detailed research studies into the holistic nature of the field effect that include epigenetic, gene expression, and comprehensive mutational analyses. Related but distinct from this is the controversial issue of multifocal origin, that is, whether cancers can arise independently from each other within a cancerized field. This issue is not one of purely academic interest as the relationship between tumor at different sites has important implications for tumor staging, treatment, margin assessment, and risk of recurrence, as leaving behind a cancerized field of predisposed cells potentially places a patient at a higher risk of recurrence.

In tumors such as high-grade serous carcinoma, it could be argued that the impact of a field change is the susceptibility of cells at different sites, for example, tubal epithelium, ovarian surface epithelium, or pelvic peritoneum, to the same driver mutation, and therefore that the presence of identical TP53 mutations does not necessarily imply a single origin. Although this is a theoretical possibility, several factors argue against this mechanism in highgrade serous carcinoma. Of the 7 mutations observed in this small series, only those seen in cases 5 and 7 are 'hot spot' mutations that arguably could occur at a high frequency sporadically. The others are relatively rare and no plausible mechanism can be advanced for their independent occurrence at two different sites. Nevertheless, this does not disprove their simultaneous occurrence because of an underlying unrecognized mutation-specific risk factor. Such a mechanism can only be demonstrated through more detailed molecular analysis for demonstration of clonal evolution. Although this was not 
the remit of the current study, this has been amply investigated in previous elegant studies. These have shown consistent results demonstrating high-grade serous carcinoma to be characterized by tremendous genomic heterogeneity that arises at a very early stage, with a variety of shared mutations other than TP53 occurring at an early stage, and mutational and copy number changes occurring subsequently. ${ }^{23,28}$ This diversity between tumor from different anatomic sites occurs in the absence of any selection pressure resulting from chemotherapy. Despite the diversity, the study of spatial and temporal evolution patterns within individual cases overwhelmingly demonstrates origin from a single clone, ${ }^{23,28}$ and

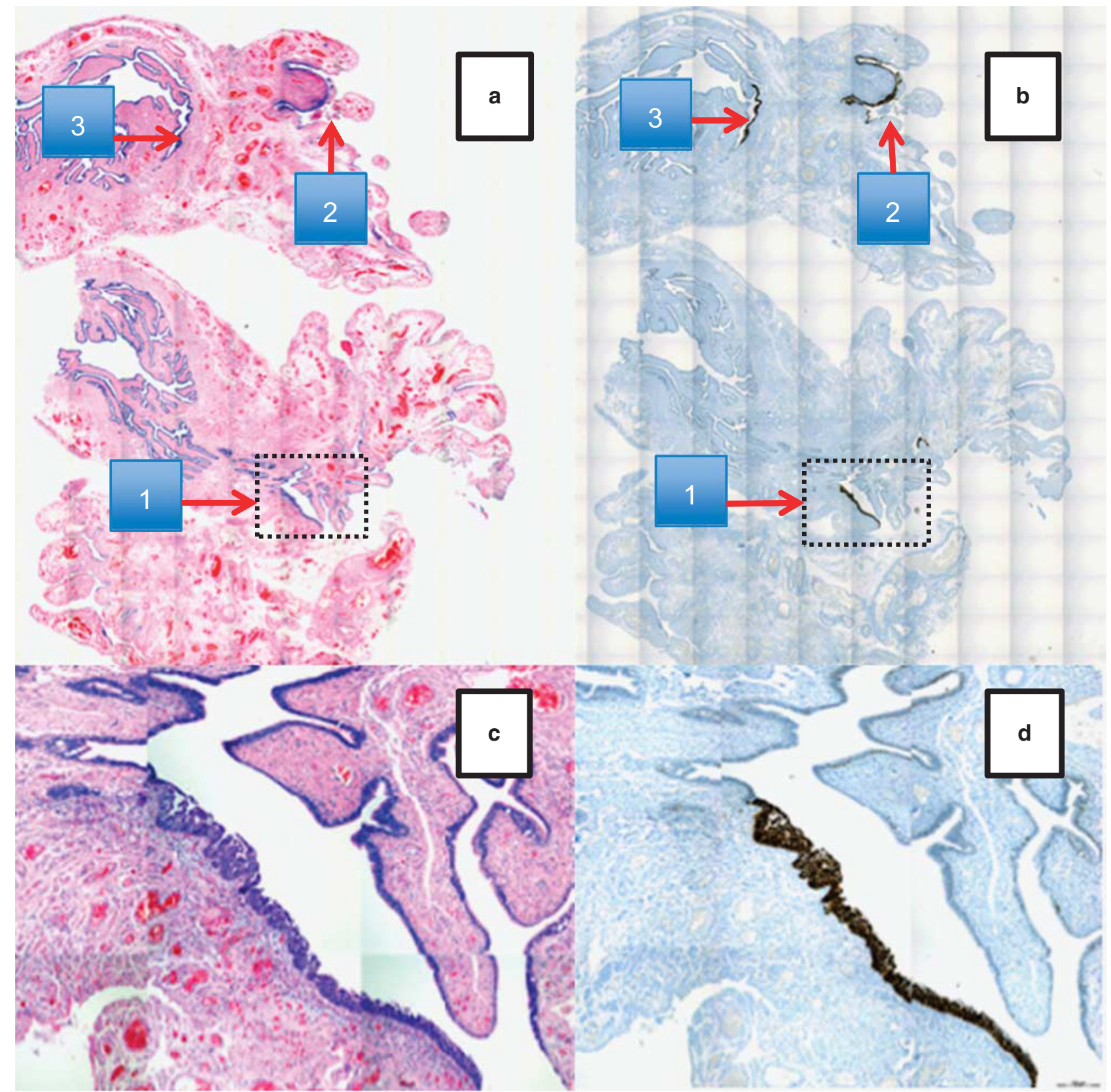

Figure 2 TP53 mutational analysis in STIC (case 7). (a, b) Hematoxylin and eosin and p53 immunostained sections of the tubal fimbria showing three discrete areas of involvement by serous tubal intraepithelial carcinoma. One area is highlighted and is shown at higher magnification in (c, d), with strong diffuse p53 immunoreactivity in the cytologically atypical cells. (e) Droplet digital PCR (ddPCR) results for the same region shown in (c, d), after laser-capture microdissection and DNA extraction. ddPCR confirms the presence of the wild-type and mutant alleles (c.742C > T) in this microdissected region of serous tubal intraepithelial carcinoma. Each dot represents a single DNA template-molecule microdroplet emulsion PCR reaction, with allele-specific reporter probe fluorescence. Mutant (FAM; Chanel 1; y axis) or wild-type (HEX; Chanel 2; $x$ axis) fluorescence are plotted in the graph and $40.5 \%$ of droplets were measured to be mutant in our lasercapture microdissected specimen. The same mutation was also demonstrated by ddPCR, at similar allelic frequency, in the other two regions of STIC seen in (a, b) (results not shown). 


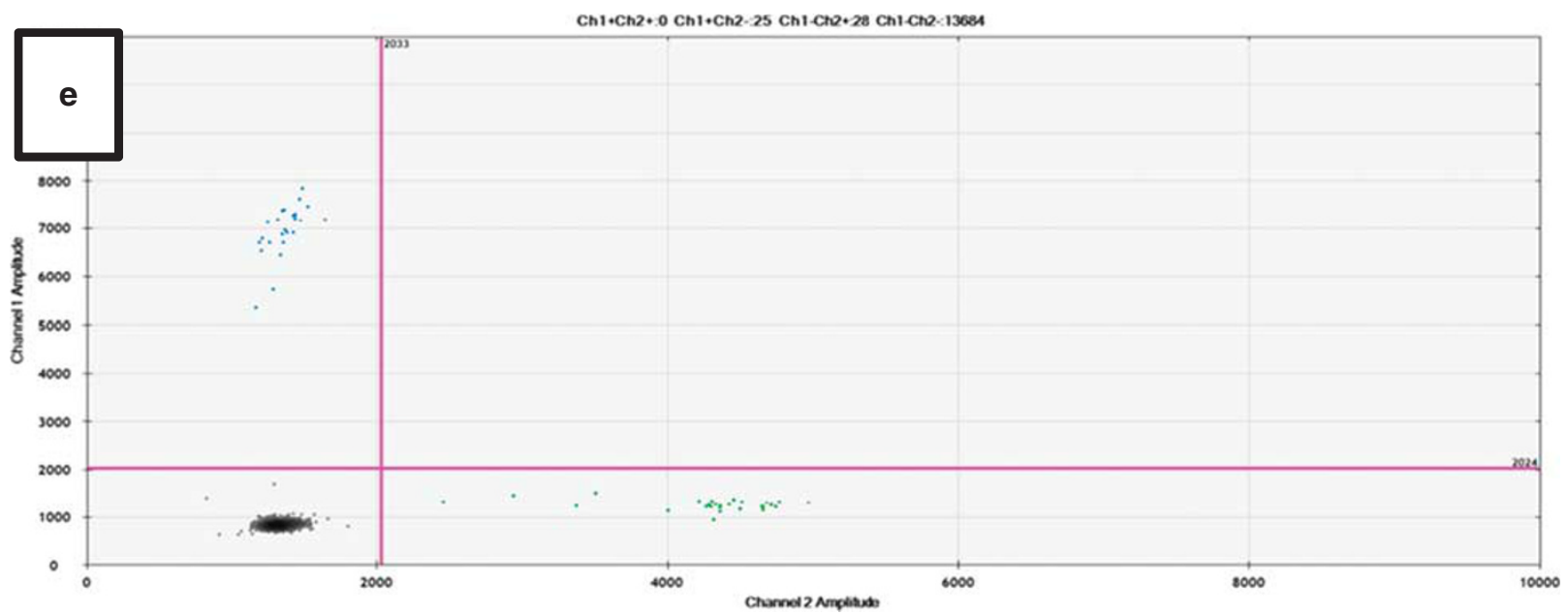

Figure 2 (Continued)

TP53 mutation is established as a ubiquitous driver event, with frequent early loss of heterozygosity and resultant loss of the normal allele giving that clone a selection advantage. ${ }^{28}$ The finding of an identical TP53 mutation in tumor at different disease sites is thereby widely accepted as valid proof of clonality and unifocal origin.

Having excluded the possibility that these are synchronous independent primary tumors, the question then arises as to which is the primary and which is the metastatic focus. This study is not able to offer a definite answer to this question. In the past, the larger size of the ovarian compared with the fallopian tube involvement would have been interpreted as evidence of the ovarian tumor being the primary (the dominant mass criterion for assignment of primary site) but it is now established that ovarian metastasis from a wide range of primary sites, for example, vermiform appendix, uterine cervix, endometrium, and stomach, can be much larger than the primary tumor and the dominant mass approach to ascertaining primary site is now discredited. As noted previously, in recent studies where extrauterine highgrade serous carcinoma was detected incidentally, in all examples of a unifocal tumor the tumor was located in the fallopian tube mucosa, providing compelling evidence that in most cases of extrauterine high-grade serous carcinoma showing both tubal and ovarian involvement or more extensive spread, the tube is likely to be the primary site. ${ }^{9-11}$

There is also molecular evidence supporting tubal origin in most extrauterine high-grade serous carcinomas. Kuhn et al ${ }^{21}$ performed mutational analyses on 29 cases of high-grade serous carcinoma and demonstrated a clonal relationship between 27 paired cases of serous tubal intraepithelial carcinoma and highgrade serous carcinoma. A study of telomere shortening in 12 paired cases of serous tubal intraepithelial carcinoma and high-grade serous carcinoma ${ }^{29}$ found a significant shortening of telomeres in serous tubal intraepithelial carcinoma lesions compared with highgrade serous carcinoma. Telomere shortening is one of the earliest events in cancer progression and short telomeres cannot support the replication rates required by invasive cancers; therefore the finding of shortened telomeres in serous tubal intraepithelial carcinoma provides evidence that this is the earlier lesion. ${ }^{30,31}$ Centrosome number abnormality and CCNE1 amplification ${ }^{32}$ was studied by the same group. CCNE1 encodes the gene for cyclin E1, the upregulation of which is thought to be an early event in malignant transformation; 33 increased cyclin E1 leads to chromosomal instability by causing aberrant centrosome duplication. ${ }^{34,35}$ They found an increase in $C C N E 1$ copy number in serous tubal intraepithelial carcinoma lesions but there were greater centrosome numbers per tumor cell in the high-grade serous carcinoma compared with serous tubal intraepithelial carcinoma, providing further evidence that serous tubal intraepithelial carcinoma precedes high-grade serous carcinoma.

In two cases in the present series there were bilateral ovarian high-grade serous carcinomas with no tubal lesion found on complete examination of both tubes with additional deeper levels, ${ }^{17}$ and one of the ovarian tumors is presumed to have been the primary neoplasm.

In the single case in this series with bilateral tubal mucosal involvement, it can be inferred that one tubal lesion is the primary tumor and the other represents metastasis. Eckert et $a l^{28}$ performed nextgeneration (exome) sequencing in eight widely disseminated sporadic extrauterine high-grade serous carcinomas with serous tubal intraepithelial carcinoma and fallopian tube, ovarian, and omental high-grade serous carcinoma. They concluded, based on genomic analysis of disease progression, that in two cases the serous tubal intraepithelial carcinoma lesions were more likely to represent metastasis to the fallopian tube and that in a small proportion of cases, serous tubal intraepithelial carcinoma lesions can be metastatic. This is certainly the case for widely disseminated disease and bilateral tubal 
mucosal tumor, that is, one is metastatic, and is not surprising as other cancers have been reported to metastasize to the fallopian tube producing lesions microscopically identical to serous tubal intraepithelial carcinoma. ${ }^{36,37}$ Interestingly, a recent report of a case of bilateral tubal high-grade serous carcinoma in a patient with a BRCA1 germline mutation showed different TP53 mutations in the two tubes, suggestive of independent tumors in this high-risk patient; ${ }^{38}$ identification of more than one TP53 mutation in a high-grade serous carcinoma has been reported in the past but this is infrequent, especially outside the setting of patients with genetic predisposition to development of high-grade serous carcinoma, that is, hereditary breast and ovarian cancer syndrome. ${ }^{6,7}$

The results presented herein have implications for FIGO staging. Using the current FIGO staging system for carcinomas of the ovary and fallopian tube, ${ }^{39}$ cases 4, 5, and 7 would be considered FIGO stage IIA, whereas cases 1-3 would be classified as stage IB. This is not rational given that all represent a primary high-grade serous carcinoma with metastasis to the other adnexal site and logically all should be considered as stage II. There is further controversy about staging of cases exemplified by case 6 (serous tubal intraepithelial carcinoma with ovarian highgrade serous carcinoma) with some classifying this as a stage I ovarian primary and others as a stage IIA tubal primary. This is largely because of differing opinions as to whether serous tubal intraepithelial carcinoma should be considered a disease site for staging purposes. In a recent international survey, a case of serous tubal intraepithelial carcinoma with invasive ovarian high-grade serous carcinoma was classified as a stage I ovarian primary and a stage II tubal primary, respectively, by $27 \%$ and $45 \%$ of pathologists. ${ }^{40}$ Among clinicians, an equal number of respondents $(37 \%)$ would stage such a case as a stage I ovarian primary and a stage II tubal primary. ${ }^{40}$ Based on our results, it appears logical to regard the ovaries and tubes (whether containing serous tubal intraepithelial carcinoma or invasive high-grade serous carcinoma) as representing two sites of disease, one primary and the other metastatic. Although the stage assignment is controversial in such patients, all would receive adjuvant chemotherapy, if medically fit, and hence treatment would not be influenced by the convention used to assign stage. Nevertheless, unless we stage these early cases consistently it will never become apparent whether these staging distinctions provide valid prognostic separation. This is particularly important as efforts toward early detection, opportunistic salpingectomy, and detailed pathological examination of the fallopian tubes are likely to result in an increase in the numbers of cases of low-stage extrauterine highgrade serous carcinoma diagnosed worldwide.

In conclusion, the consistent finding of identical TP53 mutations at both sites in this series of sporadic low-stage extrauterine high-grade serous carcinoma with only two sites of disease, located on opposite adnexa, argues strongly against multifocal origin of high-grade serous carcinoma as part of a field change. These findings should be taken into account for disease staging; we suggest that only single organconfined disease should be considered as stage I, as bilateral adnexal involvement indicates metastasis from one side to the other. We also believe that serous tubal intraepithelial carcinoma in combination with high-grade serous carcinoma at another site should be considered a disease site for the purposes of staging low-stage high-grade serous carcinoma as their clonal relationship, as established in our study, indicates the serous tubal intraepithelial carcinoma to be either the primary or a metastatic site of disease.

\section{Acknowledgments}

The authors would like to express their gratitude to Professor David Gonzalez de Castro, Professor of Molecular Pathology, Queen's University, Belfast, UK, for reviewing the results, interpretation, and final manuscript.

\section{Disclosure/conflict of interest}

The authors declare no conflict of interest.

\section{References}

1 Auersperg N, Wong AS, Choi K-C, et al. Ovarian surface epithelium: biology, endocrinology, and pathology 1 . Endocrine Rev 2001;22:255-288.

2 Pothuri B, Leitao MM, Levine DA, et al. Genetic analysis of the early natural history of epithelial ovarian carcinoma. PLoS ONE 2010;5:e10358.

3 Schorge JO, Muto MG, Lee SJ, et al. BRCA1-related papillary serous carcinoma of the peritoneum has a unique molecular pathogenesis. Cancer Res 2000;60: 1361-1364.

4 Muto MG, Welch WR, Mok SC, et al. Evidence for a multifocal origin of papillary serous carcinoma of the peritoneum. Cancer Res 1995;55:490-492.

5 Bandera CA, Muto MG, Welch WR, et al. Genetic imbalance on chromosome 17 in papillary serous carcinoma of the peritoneum. Oncogene 1998;16:3455-3459.

6 Schorge JO, Muto MG, Welch WR, et al. Molecular evidence for multifocal papillary serous carcinoma of the peritoneum in patients with germline BRCA1 mutations. J Natl Cancer Inst 1998;90:841-845.

7 Kindelberger DW, Lee Y, Miron A, et al. Intraepithelial carcinoma of the fimbria and pelvic serous carcinoma: Evidence for a causal relationship. Am J Surg Pathol 2007;31:161-169.

8 Przybycin CG, Kurman RJ, Ronnett BM, et al. Are all pelvic (nonuterine) serous carcinomas of tubal origin? Am J Surg Pathol 2010;34:1407-1416.

9 Rabban JT, Garg K, Crawford B, et al. Early detection of high-grade tubal serous carcinoma in women at low risk for hereditary breast and ovarian cancer syndrome by systematic examination of fallopian tubes incidentally removed during benign surgery. Am J Surg Pathol 2014;38:729-742. 
10 Gilks CB, Irving J, Kobel M, et al. Incidental nonuterine high-grade serous carcinomas arise in the fallopian tube in most cases: further evidence for the tubal origin of high-grade serous carcinomas. Am J Surg Pathol 2015;39:357-364.

11 Morrison JC, Blanco LZ Jr., Vang R, et al. Incidental serous tubal intraepithelial carcinoma and early invasive serous carcinoma in the nonprophylactic setting: analysis of a case series. Am J Surg Pathol 2015;39: 442-453.

12 Singh N, Gilks CB, Wilkinson N, et al. Assignment of primary site in high-grade serous tubal, ovarian and peritoneal carcinoma: a proposal. Histopathology 2014;65:149-154.

13 Singh N, Gilks CB, Wilkinson N, et al. The secondary Mullerian system, field effect, BRCA, and tubal fimbria: our evolving understanding of the origin of tubo-ovarian high-grade serous carcinoma and why assignment of primary site matters. Pathology 2015;47:423-431.

14 Singh N, Gilks CB, Hirschowitz L, et al. Primary site assignment in tubo-ovarian high-grade serous carcinoma: consensus statement on unifying practice worldwide. Gynecol Oncol 2016;141:195-198.

15 Singh N, Gilks CB, Hirshowitz L, et al. Adopting a uniform approach to site assignment in tubo-ovarian high-grade serous carcinoma: the time has come. Int J Gynecol Pathol 2016;35:230-237.

16 McCluggage WG, Judge MJ, Clarke BA, et al. Data set for reporting of ovary, fallopian tube and primary peritoneal carcinoma: recommendations from the International Collaboration on Cancer Reporting (ICCR). Mod Pathol 2015;28:1101-1122.

17 Singh N, Benson JL, Gan C, et al. Disease distribution in low-stage tubo-ovarian high-grade serous carcinoma (HGSC): implications for assigning primary site and FIGO stage. Int J Gynecol Pathol; advance online publication, 29 April 2017; doi: 10.1097/ PGP.0000000000000392 [e-pub ahead of print].

18 McConechy MK, Talhouk A, Leung S, et al. Endometrial carcinomas with POLE exonuclease domain mutations have a favorable prognosis. Clin Cancer Res 2016;22:2865-2873.

19 Carlson JW, Miron A, Jarboe EA, et al. Serous tubal intraepithelial carcinoma: its potential role in primary peritoneal serous carcinoma and serous cancer prevention. J Clin Oncol 2008;26:4160-4165.

20 Bijron JG, Seldenrijk CA, Zweemer RP, et al. Fallopian tube intraluminal tumor spread from noninvasive precursor lesions: a novel metastatic route in early pelvic carcinogenesis. Am J Surg Pathol 2013;37: 1123-1130.

21 Kuhn E, Kurman RJ, Vang R, et al. TP53 mutations in serous tubal intraepithelial carcinoma and concurrent pelvic high-grade serous carcinoma-evidence supporting the clonal relationship of the two lesions. J Pathol 2012;226:421-426.

22 McDaniel AS, Stall JN, Hovelson DH, et al. Nextgeneration sequencing of tubal intraepithelial carcinomas. JAMA Oncol 2015;1:1128-1132.

23 Bashashati A, Ha G, Tone A, et al. Distinct evolutionary trajectories of primary high-grade serous ovarian cancers revealed through spatial mutational profiling. J Pathol 2013;231:21-34.
24 Kobel M, Piskorz AM, Lee S, et al. Optimized p53 immunohistochemistry is an accurate predictor of TP53 mutation in ovarian carcinoma. J Pathol Clin Res 2016;2:247-258.

25 Cancer Genome Atlas Research Network. Integrated genomic analyses of ovarian carcinoma. Nature 2011;474:609-615.

26 Dakubo GD, Jakupciak JP, Birch-Machin MA, et al. Clinical implications and utility of field cancerization. Cancer Cell Int 2007;7:2.

27 Chai H, Brown RE. Field effect in cancer-an update. Ann Clin Lab Sci 2009;39:331-337.

28 Eckert MA, Pan S, Hernandez KM, et al. Genomics of ovarian cancer progression reveals diverse metastatic trajectories including intraepithelial metastasis to the fallopian tube. Cancer Discov 2016;6:1342-1351.

29 Kuhn E, Meeker A, Wang T-L, et al. Shortened telomeres in serous tubal intraepithelial carcinoma: an early event in ovarian high-grade serous carcinogenesis. Am J Surg Pathol 2010;34:829.

30 Hanahan D, Weinberg RA. The hallmarks of cancer. Cell 2000;100:57-70.

31 Hanahan D, Weinberg RA. Hallmarks of cancer: the next generation. Cell 2011;144:646-674.

32 Kuhn E, Wang T-L, Doberstein K, et al. CCNE1 amplification and centrosome number abnormality in serous tubal intraepithelial carcinoma: further evidence supporting its role as a precursor of ovarian high-grade serous carcinoma. Mod Pathol 2016;29:1254-1261.

33 Karst AM, Jones PM, Vena N, et al. Cyclin E1 deregulation occurs early in secretory cell transformation to promote formation of fallopian tube-derived high-grade serous ovarian cancers. Cancer Res 2014;74: 1141-1152.

34 Fukasawa K. Centrosome amplification, chromosome instability and cancer development. Cancer Lett 2005;230:6-19.

35 Nigg EA, Stearns T. The centrosome cycle: centriole biogenesis, duplication and inherent asymmetries. Nat Cell Biol 2011;13:1154-1160.

36 Rabban JT, Vohra P, Zaloudek CJ. Nongynecologic metastases to fallopian tube mucosa: a potential mimic of tubal high-grade serous carcinoma and benign tubal mucinous metaplasia or nonmucinous hyperplasia. Am J Surg Pathol 2015;39:35-51.

37 Kommoss F, Faruqi A, Gilks CB, et al. Uterine serous carcinomas frequently metastasize to the fallopian tube and can mimic serous tubal intraepithelial carcinoma. Am J Surg Pathol 2017;41:161-170.

38 Gurda GT, Serdy KM, Yatsenko SA, et al. Synchronous bilateral tubal serous carcinomas in a patient with exon 13 duplication and loss of function of BRCA1. Int J Gynecol Pathol; advance online publication, 29 April 2017; doi: 10.1097/PGP.0000000000000392 [e-pub ahead of print].

39 Prat JFIGO Committee on Gynecologic Oncology. Staging classification for cancer of the ovary, fallopian tube, and peritoneum. Int J Gynecol Obstet 2014;124:1-5.

40 McCluggage WG, Hirschowitz L, Gilks CB, et al. The fallopian tube origin and primary site assignment in extrauterine high-grade serous carcinoma: findings of a survey of pathologists and clinicians. Int J Gynecol Pathol 2017;36:230-239.

Supplementary Information accompanies the paper on Modern Pathology website (http://www.nature.com/ modpathol) 